\title{
On the Separability of Stochastic Geometric Objects, with Applications
}

\author{
Jie Xue ${ }^{1}$, Yuan $\mathrm{Li}^{2}$, and Ravi Janardan ${ }^{3}$
}

1 Department of Computer Science and Engineering, University of Minnesota Twin Cities, 4-192 Keller Hall, 200 Union St. SE, Minneapolis, MN 55455, USA xuexx193@umn.edu

2 Department of Computer Science and Engineering, University of Minnesota Twin Cities, 4-192 Keller Hall, 200 Union St. SE, Minneapolis, MN 55455, USA lixx2100@umn.edu

3 Department of Computer Science and Engineering, University of Minnesota Twin Cities, 4-192 Keller Hall, 200 Union St. SE, Minneapolis, MN 55455, USA

janardan@umn.edu

\begin{abstract}
In this paper, we study the linear separability problem for stochastic geometric objects under the well-known unipoint/multipoint uncertainty models. Let $S=S_{R} \cup S_{B}$ be a given set of stochastic bichromatic points, and define $n=\min \left\{\left|S_{R}\right|,\left|S_{B}\right|\right\}$ and $N=\max \left\{\left|S_{R}\right|,\left|S_{B}\right|\right\}$. We show that the separable-probability (SP) of $S$ can be computed in $O\left(n N^{d-1}\right)$ time for $d \geq 3$ and $O\left(\min \left\{n N \log N, N^{2}\right\}\right)$ time for $d=2$, while the expected separation-margin (ESM) of $S$ can be computed in $O\left(n N^{d}\right)$ time for $d \geq 2$. In addition, we give an $\Omega\left(n N^{d-1}\right)$ witness-based lower bound for computing SP, which implies the optimality of our algorithm among all those in this category. Also, a hardness result for computing ESM is given to show the difficulty of further improving our algorithm. As an extension, we generalize the same problems from points to general geometric objects, i.e., polytopes and/or balls, and extend our algorithms to solve the generalized SP and ESM problems in $O\left(n N^{d}\right)$ and $O\left(n N^{d+1}\right)$ time, respectively. Finally, we present some applications of our algorithms to stochastic convex-hull related problems.
\end{abstract}

1998 ACM Subject Classification F.2.2 Nonnumerical Algorithms and Problems - Geometrical problems and computations

Keywords and phrases Stochastic objects, linear separability, separable-probability, expected separation-margin, convex hull

Digital Object Identifier 10.4230/LIPIcs.SoCG.2016.62

\section{Introduction}

Linear separability describes the property that a set of $d$-dimensional bichromatic (red and blue) points can be separated by a hyperplane such that all the red points lie on one side of the hyperplane and all the blue points lie on the other side. This problem has been well studied for years in computational geometry, and is widely used in machine learning and data mining for data classification. However, existing linear-separation algorithms require that all the input points must have fixed locations, which is rarely true in reality due to imprecise sampling from GPS, robotics sensors, or some other probabilistic systems. It is therefore essential to study the conventional linear separability problem under uncertainty.

(c) (i) () Jie Xue, Yuan Li, and Ravi Janardan;

32nd International Symposium on Computational Geometry (SoCG 2016).

Editors: Sándor Fekete and Anna Lubiw; Article No. 62; pp. 62:1-62:16

Leibniz International Proceedings in Informatics 
In this paper, we study the linear separability problem under two different uncertainty models, i.e., the unipoint and multipoint models [4]. In the former, each stochastic data point has a fixed and known location, and has a positive probability to exist at that location; whereas in the latter, each stochastic data point occurs in one of discretely-many possible locations with known probabilities, and the existence probabilities of each point sum up to at most 1 to allow for its absence. Our focus is to compute the separable-probability (SP) and the expected separation-margin (ESM) for a given set of bichromatic stochastic points (or general geometric objects) in $\mathbb{R}^{d}$ for $d \geq 2$, where the former is the probability that the existent points (or objects) are linearly separable, and the latter is the expectation of the separation-margin of the existent points (or objects). (See Section 3.1 for a detailed and formal definition of the latter.)

The main contributions of this paper are:

1. We propose an $O\left(n N^{d-1}\right)$-time algorithm, which uses linear space, for solving the SP problem when given a set of bichromatic stochastic points in $\mathbb{R}^{d}$ for $d \geq 3$. (The runtime is $O\left(\min \left\{N^{2}, n N \log N\right\}\right)$ for $d=2$.) We also show an $\Omega\left(n N^{d-1}\right)$ lower bound for all witness-based algorithms, which implies the optimality of our algorithm among all witness-based methods for $d \geq 3$. (See Section 2.)

2. We show that the ESM of the above dataset can be computed in $O\left(n N^{d}\right)$ time for $d \geq 2$, using linear space. A hardness result is also given to show the total number of distinct possible separation-margins is $\Theta\left(n N^{d}\right)$, which implies that it may be difficult to achieve a better runtime. (See Section 3.)

3. We extend our algorithms to compute the SP and the ESM for datasets containing general stochastic geometric objects, such as polytopes and/or balls. Our generalized algorithms solve the former problem in $O\left(n N^{d}\right)$ time, and the latter in $O\left(n N^{d+1}\right)$ time, using linear space. (See Section 4.)

4. We provide some applications of our algorithms to stochastic convex-hull (SCH) related problems. Specifically, by taking advantage of our SP algorithm, we give a method to compute the SCH membership probability, which matches the best known bound but is more direct. Also, we consider some generalized versions of this problem and show how to apply our separability algorithms to solve them efficiently. (See Section 5.)

Due to space limitations, most proofs and some details are omitted here, but can be found in the full version [18].

\subsection{Related work}

The study of computational geometry problems under uncertainty is a relatively new topic, and has attracted a lot of attention; see [5] and [7] for two surveys. Different uncertainty models and related problems have been investigated in recent years. See $[1,2,3,8,14,15,19]$ for example. The unipoint/multipoint uncertainty model, which we use in this paper, was first defined in $[4,17]$, and has been applied in many recent papers. For instance, in [12], Kamousi et al. studied the stochastic minimum spanning tree problem, and computed its expected length. Suri et al. investigated the most likely convex hull problem over uncertain data in [17]; the similar topic was revisited by Agarwal et al. in [4] to compute the probability that a query point is inside the uncertain hull. In [16], Suri and Verbeek studied the most likely Voronoi Diagram (LVD) in $\mathbb{R}^{1}$ under the unipoint model, and the expected complexity of LVD was further improved by Li et al. in [13], who explored the stochastic line arrangement problem in $\mathbb{R}^{2}$. In [6], Agrawal et al. proposed efficient algorithms for the most likely skyline problem in $\mathbb{R}^{2}$ and gave NP-hardness results in higher dimensions. 
Recently, in [8], de Berg et al. studied the separability problem given a set of bichromatic imprecise points in $\mathbb{R}^{2}$ in a setting that each point is drawn from an imprecision region.

Very recently, in an unpublished manuscript [11], one of our proposed problems, the SP computing problem, was independently studied by Fink et al. under the same uncertainty model, and similar results were obtained, i.e., an $O\left((n+N)^{d}\right)=O\left(N^{d}\right)$-time and $O(n+N)=$ $O(N)$-space algorithm for computing the SP of a given set of bichromatic stochastic points in $\mathbb{R}^{d}$. In fact, before the final step of the improvement, the time bound achieved was $O\left(N^{d} \log N\right)$, and then duality [9] and topological sweep [10] techniques were applied to eliminate the $\log$ factor. On the other hand, our algorithm runs initially in $O\left(n N^{d-1} \log N\right)$ time using linear space, and can be further improved to $O\left(n N^{d-1}\right)$ runtime by using the same techniques. (A careful discussion will be given in Section 2.) Note that the algorithm in [11] always runs in $\Theta\left(N^{d}\right)$ time (no matter how small $n$ is) and, more importantly, this runtime appears to be intrinsic: all possible $d$-tuples of (distinct) points have to be enumerated in order to correctly compute the SP. Our time bound matches the bound in [11] when $n=\Theta(N)$. However, when $n \ll N$, our method is much faster, and, in fact, this is usually the case in many real classification problems in machine learning and data mining.

In terms of how to solve the problem, Fink et al.'s method is very different from ours. Their computation of SP relies on an additional dummy anchor point, and based on this point, the probability is computed in an inclusion-exclusion manner. On the other hand, our method solves the problem more directly: it does not introduce any additional points and the SP is eventually computed using a simple addition principle. Furthermore, our algorithm can be easily extended to solve many generalized problems (e.g., multiple colors, general geometric objects, etc.)

\subsection{Basic notations and preliminaries}

Throughout this paper, the basic notations we use are the following. We use $S=S_{R} \cup S_{B}$ to denote the given stochastic bichromatic dataset, where $S_{R}$ (resp. $S_{B}$ ) is a set of red (resp. blue) stochastic points (or general geometric objects in Section 4 ). The notations $n$ and $N$ are used to denote the sizes of the smaller and larger classes of $S$ respectively, i.e., $n=\min \left\{\left|S_{R}\right|,\left|S_{B}\right|\right\}$ and $N=\max \left\{\left|S_{R}\right|,\left|S_{B}\right|\right\}$, and $d$ is used to denote the dimension. In this paper, we always assume that $d$ is a constant. When we need to denote a normal bichromatic dataset (without considering the existence probabilities), we usually use the notation $T=T_{R} \cup T_{B}$. The coordinates of a point $x \in \mathbb{R}^{d}$ are denoted as $x^{(1)}, x^{(2)}, \ldots, x^{(d)}$. If $T$ is a dataset in $\mathbb{R}^{d}$ and $U$ is some linear subspace of $\mathbb{R}^{d}$, we use $T^{U}$ to denote a new dataset in the space $U$, which is obtained by orthogonally projecting $T$ from $\mathbb{R}^{d}$ onto $U$.

We say a set of bichromatic points is strongly separable iff there exists a hyperplane, $h$, so that all the red points strictly lie on one side of $h$ while all the blue points strictly lie on the other side. Also, we can define the concept of weakly separable similarly, except that we allow points to lie on the hyperplane. A hyperplane that strongly (resp., weakly) separates a set of bichromatic points is called a strong (resp., weak) separator. The following is a fundamental result we will use in various places of the paper.

Theorem 1. Suppose $T=T_{R} \cup T_{B}$ is a set of bichromatic points in $\mathbb{R}^{d}$. $T$ is strongly separable by a hyperplane iff $\mathcal{C H}\left(T_{R}\right) \cap \mathcal{C H}\left(T_{B}\right)=\emptyset$, where $\mathcal{C H}(\cdot)$ denote the convex hull of the indicated point-set. 


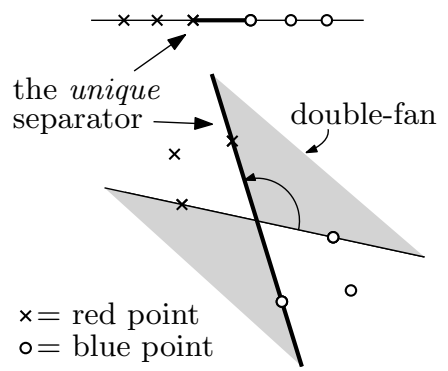

Figure 1 Illustrating the unique separator we choose for separable instances in $\mathbb{R}^{1}$ and $\mathbb{R}^{2}$.

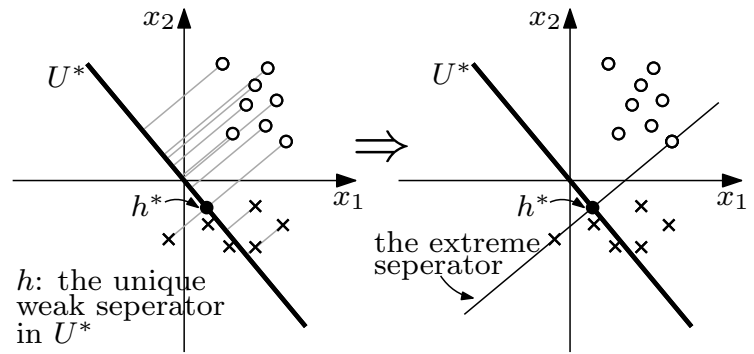

Figure 2 Illustrating the extreme separator in $\mathbb{R}^{2}$.

\section{Separable-probability}

The separable-probability (SP) of a stochastic bichromatic dataset $S=S_{R} \cup S_{B}$ in $\mathbb{R}^{d}$ refers to the probability that the existent points in $S$ (obtained by a random experiment) are (strongly) separable. For simplicity, we describe here the details of our algorithm under the unipoint model only. The generalization of our algorithm to the multipoint model is quite straightforward and can be found in the full version [18].

Trivially, given a dataset $S$, its SP can be computed by simply enumerating all the $2^{|S|}$ possible instances of $S$ and summing up the probabilities of the separable ones, which takes exponential time. In order to solve the problem more efficiently than by brute-force, one has to categorize all the separable instances of $S$ into a reasonable number of groups such that the sum of the probabilities of the instances in each group can be easily computed. A natural approach is to charge each separable instance to a unique separator, and use that as the key to do the grouping. The uniqueness requirement here is to avoid over-counting. In addition, all these separators should be easy to enumerate and the sum of the probabilities of those separable instances charged to each separator should be efficiently computable. In $\mathbb{R}^{1}$ and $\mathbb{R}^{2}$, this is easy to achieve. For example, in $\mathbb{R}^{1}$, given a separable instance, all the possible separators form a segment, and we can choose the leftmost endpoint as the unique separator; in $\mathbb{R}^{2}$, all the possible separators of a separable instance form a double-fan, and we can choose the most counterclockwise one, which goes through exactly one red and one blue point, as the unique separator. (See Figure 1 for an illustration.) It is easy to see that, with the separators chosen above, the SP of the stochastic dataset can be easily computed by considering the sum of the probabilities of the instances charged to each such separator. However, to define such a separator for a separable instance beyond $\mathbb{R}^{2}$ turns out to be hard and challenging. To solve this problem, we define an important concept called extreme separator in Section 2.1, and apply this concept to compute the SP of $S$ in Section 2.2.

For convenience, we assume that the points given in $S$ have the strong general position property (SGPP), which is defined as follows. Let $I=\left\{i_{1}, i_{2}, \ldots, i_{|I|}\right\}$ be any subset of 


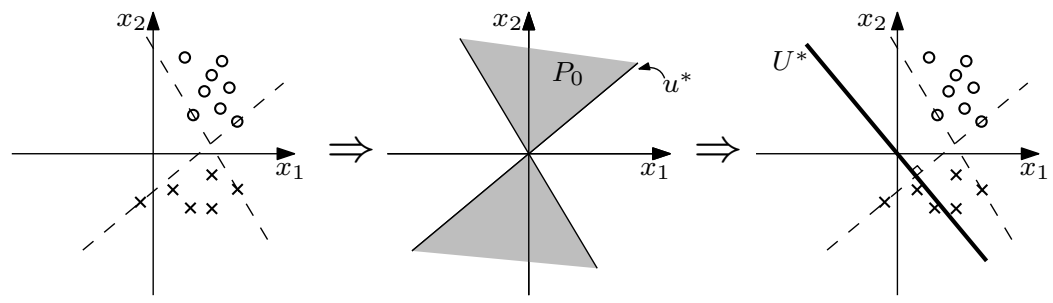

Figure 3 Illustrating $U^{*}$ in $\mathbb{R}^{2}$. Note that $P_{1}$ is not shown to avoid confusion.

the index set $\{1,2, \ldots, d\}$ where $i_{1}<i_{2}<\cdots<i_{|I|}$. We define a projection function $\phi_{I}: \mathbb{R}^{d} \rightarrow \mathbb{R}^{|I|}$ as $\phi_{I}(x)=\left(x^{\left(i_{1}\right)}, x^{\left(i_{2}\right)}, \ldots, x^{\left(i_{|I|}\right)}\right)$. Also, for any $X \subseteq \mathbb{R}^{d}$, we define $\Phi_{I}(X)=\left\{\phi_{I}(x): x \in X\right\}$.

Let $T$ be a set of points in $\mathbb{R}^{d}$. When $d \leq 2$, we say $T$ has SGPP iff it is in general (linear) position, i.e., affinely independent. When $d \geq 3$, we say $T$ has SGPP iff

1. $T$ is in general (linear) position;

2. $\Phi_{J}(T)$ has SGPP for $J=\{3,4, \ldots, d\}$.

- Remark. This assumption is actually not stronger than the conventional general position assumption, since one can easily apply linear transformations to make a set of points in general position have SGPP (the separability of a dataset is invariant under non-singular linear transformations). See the full version [18] for a detailed algorithm to achieve this.

\subsection{Extreme separator}

To solve the SP problem, we define a very important concept called extreme separator through a sequence of steps. Suppose a separable bichromatic dataset $T=T_{R} \cup T_{B}$ with SGPP is given in $\mathbb{R}^{d}(d \geq 2)$. Assume that both $T_{R}$ and $T_{B}$ are nonempty. Let $\mathcal{V}$ be the collection of the $(d-1)$-dim linear subspaces of $\mathbb{R}^{d}$ whose equation is of the form $a x_{1}+b x_{2}=0$, where $a$ and $b$ are constants not equal to 0 simultaneously. In other words, $\mathcal{V}$ contains all the $(d-1)$-dim linear subspaces that are perpendicular to the $x_{1} x_{2}$-plane and go through the origin. Then there is a natural one-to-one correspondence between $\mathcal{V}$ and $\mathbb{P}^{1}$ (the 1-dim projective space), $\sigma:\left[a x_{1}+b x_{2}=0\right] \longleftrightarrow[a: b]$. For convenience, we use $\sigma$ to denote the maps in both directions in the rest of this paper. We now define a map $\pi_{T}: \mathcal{V} \rightarrow\{0,1\}$ as follows. For any $V \in \mathcal{V}$, we orthogonally project all the points in $T$ onto $V$ and use $T^{V}=T_{R}^{V} \cup T_{B}^{V}$ to denote the new dataset after projection. If $T^{V}$ is strongly separable, we set $\pi_{T}(V)=1$. Otherwise, we set $\pi_{T}(V)=0$. The map $\pi_{T}$ induces another map $\pi_{T}^{*}: \mathbb{P}^{1} \rightarrow\{0,1\}$ by composing with the correspondence $\sigma$. Let $P_{0}$ and $P_{1}$ be the pre-images of $\{0\}$ and $\{1\}$ under $\pi_{T}^{*}$, respectively (see Figure 3). By applying Theorem 1, it is easy to prove the following.

- Theorem 2. $P_{0}$ is a connected closed subspace of $\mathbb{P}^{1} . P_{0}=\emptyset$ iff $\Phi_{J}(T)$ is strongly separable in $\mathbb{R}^{d-2}$ for $J=\{3,4, \ldots, d\}$.

We now have two cases, i.e., $P_{0} \neq \emptyset$ and $P_{0}=\emptyset$. If $P_{0} \neq \emptyset$, we define the extreme separator of $T$ as follows. Since $P_{0}$ is a connected closed subspace of $\mathbb{P}^{1}$, it has a unique clockwise boundary point $u^{*}$ (i.e., $u^{*}$ is the last point of $P_{0}$ in the clockwise direction). Let $U^{*}=\sigma\left(u^{*}\right)$ be the linear subspace in $\mathcal{V}$ corresponding to $u^{*}$ (see Figure 3 again). The following theorem reveals the separability property of $T^{U^{*}}$.

Theorem 3. $T^{U^{*}}$ is weakly separable and there exists only one weak separator. Furthermore, the unique separator of $T^{U^{*}}$ goes through exactly $d$ points, of which at least one is in $T_{R}^{U^{*}}$ and one is in $T_{B}^{U^{*}}$. 
- Definition 4 (Derived Separator). Let $U$ be a $k$-dim linear subspace $(k<d)$ of $\mathbb{R}^{d}$. Suppose $h$ is a strong (resp., weak) separator of $T^{U}$ in the space $U$. It is easy to see that the pre-image, $h^{\prime}$, of $h$ under the orthogonal projection $\mathbb{R}^{d} \rightarrow U$ is a strong (resp., weak) separator of $T$ in $\mathbb{R}^{d}$. We call $h^{\prime}$ the derived separator of $h$ in $\mathbb{R}^{d}$.

Let $h^{*}$ be the unique weak separator of $T^{U^{*}}$. We define the extreme separator of $T$ as the derived separator of $h^{*}$ in $\mathbb{R}^{d}$. (See Figure 2.) At the same time, we call $U^{*}$ the auxiliary subspace defining the extreme separator. Clearly, the extreme separator and the auxiliary subspace are perpendicular to each other.

On the other hand, if $P_{0}=\emptyset$, we recursively define the extreme separator of $T$ as the derived separator of the extreme separator of $\Phi_{J}(T)$, for $J=\{3,4, \ldots, d\}$. Note that $P_{0}$ is nonempty when $d=2$. To complete this recursive definition, we define the extreme separator in $\mathbb{R}^{1}$ as the weak separator (which is a point) with the smallest coordinate.

Note that the above definition of the extreme separator is only for the case that both $T_{R}$ and $T_{B}$ are nonempty. In the trivial case where $T_{R}$ and/or $T_{B}$ is empty, we simply define the extreme separator as $x_{d}=\infty$.

To understand the intuition for the extreme separator, let us consider the case $d=3$. Imagine there is a plane rotating clockwise around the $x_{3}$-axis. We keep projecting the points in $T$ (orthogonally) to that plane and track the separability of the projection images. If the images are always separable, then the extreme separator is defined recursively. Otherwise, there is a closed period of time in which the images are inseparable, which is subsequently followed by an open period in which the images are separable. At the connection of the two periods (from the inseparable one to the separable one), the images are weakly separable by a unique weak separator. Then the rotating plane at this point is just the auxiliary subspace, and the extreme separator is obtained by orthogonally "extending" the unique weak separator to $\mathbb{R}^{3}$.

\subsection{Computing the separable-probability}

We remind the reader that $n=\min \left\{\left|S_{R}\right|,\left|S_{B}\right|\right\}$ and $N=\max \left\{\left|S_{R}\right|,\left|S_{B}\right|\right\}$. Set $J=$ $\{3,4, \ldots, d\}$. If the existent points in $S$ are separable, then there are two cases: 1) the extreme separator of the existent points is defined recursively (the case of $P_{0}=\emptyset$ ) or equal to $x_{d}=\infty$ (the trivial case); 2) the extreme separator of the existent points is directly defined in $\mathbb{R}^{d}$ (the case of $P_{0} \neq \emptyset$ ). These two cases are clearly disjoint so that the SP can be computed as the sum of their probabilities. By applying Theorem 2, the probability of the first case is equal to the SP of $\Phi_{J}(S)$. In the second case, according to Theorem 3 , the extreme separator goes through exactly $d$ points (of which at least one is red and one is blue). Thus, the SP of $S$ can be computed as

$$
\operatorname{Sep}(S)=\operatorname{Sep}\left(\Phi_{J}(S)\right)+\sum_{h \in H_{S}} \tau_{S}(h),
$$

where $H_{S}$ is the set of the hyperplanes that go through exactly $d$ points (of which at least one is red and one is blue) in $S$ and, for $h \in H_{S}, \tau_{S}(h)$ is the probability that the extreme separator of the existent points is $h$.

Clearly, for each $h \in H_{S}$, there is a unique element $U^{*} \in \mathcal{V}$ perpendicular to it ( $h$ can never be parallel to the $x_{1} x_{2}$-plane due to the SGPP of $S$ ). If $h$ is indeed the extreme separator of the existent points, then $U^{*}$ must be the auxiliary subspace. Let $E=E_{R} \cup E_{B}$ be the set of the points on $h$. In order to compute $\tau_{S}(h)$, we investigate the conditions for $h$ to be the extreme separator of the existent points. First, as the $d$ points on $h$, the points 


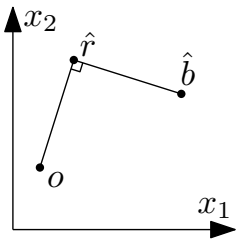

Figure 4 Illustrating the location of $o$. The space in the figure is the 2-dim subspace of $\mathbb{R}^{d}$ that is parallel to the $x_{1} x_{2}$-plane and contains $\hat{r}, \hat{b}$.

in $E$ must exist. Second, because the existent points should be weakly (but not strongly) separable after being projected onto $U^{*}$, there must exist $\hat{r} \in \mathcal{C H}\left(E_{R}\right)$ and $\hat{b} \in \mathcal{C H}\left(E_{B}\right)$ whose projection images on $U^{*}$ coincide, according to Theorem 1. (Actually, such $\hat{r}$ and $\hat{b}$ are unique if they exist, due to the SGPP of $S$.) Finally, since the extreme separator should weakly separate the existent points, all the red existent points must lie on one side of $h$ while all the blue ones must lie on the other side, except the points in $E$. Also, the side for red/blue points is specific, as $\sigma\left(U^{*}\right)$ must be the clockwise boundary of $P_{0}$. To distinguish the red/blue side of $h$, we define, based on $\hat{r}$ and $\hat{b}$, an indicator $o=\left(o^{(1)}, o^{(2)}, \ldots, o^{(d)}\right)$, where

$$
\begin{aligned}
& o^{(1)}=\hat{r}^{(1)}+\left(\hat{b}^{(2)}-\hat{r}^{(2)}\right), \\
& o^{(2)}=\hat{r}^{(2)}+\left(\hat{r}^{(1)}-\hat{b}^{(1)}\right), \\
& o^{(i)}=\hat{r}^{(i)}=\hat{b}^{(i)} \text { for } i \in J .
\end{aligned}
$$

(See Figure 4 for the location of $o$.) It is easy to see that, when all the red (resp., blue) points appear on the same (resp., opposite) side of $h$ w.r.t. $o, \sigma\left(U^{*}\right)$ is the clockwise boundary of $P_{0}$. In sum, $h$ is the extreme separator of the existent points iff

1. all the points in $E$ exist;

2. there are $\hat{r} \in \mathcal{C H}\left(E_{R}\right)$ and $\hat{b} \in \mathcal{C H}\left(E_{B}\right)$ such that their projection images on $U^{*}$ coincide;

3. no red (resp. blue) point on the opposite (resp. same) side of $h$ w.r.t. $o$ exists.

Among the three conditions, the second one has nothing to do with the existences of the stochastic points in $S$ and can be verified in constant time. If $h$ violates this condition, then $\tau_{S}(h)=0$. Otherwise, $\tau_{S}(h)$ is just equal to the product of the existence probabilities of the points in $E$ and the non-existence probabilities of the points which should not exist due to the third condition. The simplest way to compute it is to scan every point in $S$ once, which takes linear time. This then leads to an $O\left(n N^{d}\right)$ overall time for computing the SP of $S$, since $\left|H_{S}\right|$ is bounded by $O\left(n N^{d-1}\right)$.

By applying the idea of of radial-order sort in [4], we are able to reduce the runtime to $O\left(n N^{d-1} \log N\right)$. Furthermore, inspired by [11], it is possible to further eliminate the log factor in the time bound by taking advantage of duality [9] and topological sweep [10] techniques. The time complexity of our algorithm is then improved to $O\left(n N^{d-1}\right)$ for $d \geq 3$ and $O\left(\min \left\{n N \log N, N^{2}\right\}\right)$ for $d=2$. See the full version [18] for details.

\subsection{Witness-based lower bound for computing separable-probability}

When solving the SP problem, the key idea of our algorithm is to group the probabilities of those separable instances that share the same extreme separator so that the SP can be efficiently computed by considering the extreme separators instead of single instances. Actually, by extending and abstracting this idea, we are able to get a general framework for computing SP, which we call the witness-based framework. Let $S$ be the given stochastic dataset and $\mathcal{I}_{S}$ be the set of all the separable instances of $S$. The witness-based framework for computing the SP of $S$ is the following. (Here, $\mathcal{P}(\cdot)$ denotes the powerset function.) 
1. Define a set $W=\left\{h_{1}, \ldots, h_{m}\right\}$ of hyperplanes (called witness separators) with specified weights $w_{1}, \ldots, w_{m}$ and an implicitly specified witness rule $f: W \rightarrow \mathcal{P}\left(\mathcal{I}_{S}\right)$ such that

- the instances in $f\left(h_{i}\right)$ are (either strongly or weakly) separated by $h_{i}$;

- the witness probability (see Step $\mathbf{2}$ below) of each $h_{i}$ is efficiently computable;

- any instance $I \in \mathcal{I}_{S}$ satisfies $\sum_{\forall i\left(I \in f\left(h_{i}\right)\right)} w_{i}=1$.

We say the witness separator $h_{i}$ witnesses the instances in $f\left(h_{i}\right)$.

2. Compute efficiently the witness probability of each $h_{i} \in W$, which is defined as

$$
\text { witP }\left(h_{i}\right)=\sum_{I \in f\left(h_{i}\right)} \operatorname{Pr}(I),
$$

i.e., the sum of the probabilities of all the instances witnessed by $h_{i}$.

3. Compute the SP of $S$ by linearly combining the witness probabilities with the specified weights, i.e.,

$$
\operatorname{Sep}(S)=\sum_{i=1}^{m}\left(w_{i} \cdot w i t P\left(h_{i}\right)\right)=\sum_{I \in \mathcal{I}_{S}} \operatorname{Pr}(I) .
$$

Note that the witness-based framework is very general. The ways of defining witness separators and specifying witness rules may vary a lot among different witness-based algorithms. Our algorithm and the one introduced in [11], which are the only two known algorithms for computing SP at this time, both belong to the witness-based framework. Similar frameworks are also used to solve other probability-computing problems. For example, the two algorithms in [4] for computing convex-hull membership probability are both implemented by defining witness edges/facets and summing up the witness probabilities. To our best knowledge, up to now, most probability-computing problems under unipoint/multipoint model are solved by applying ideas close to this framework.

Now we show that any SP computing algorithm following the witness-based framework takes at least $\Omega\left(n N^{d-1}\right)$ time in the worst case, and thus our algorithm is optimal among this category of algorithms for any $d \geq 3$. Clearly, the runtime of a witness-based algorithm is at least $|W|=m$, i.e., the number of the witness separators. Then a question naturally arises: how many witness separators do we need for computing SP? From the above framework, one restriction for $W$ is that each separable instance of $S$ must be witnessed by at least one witness separator $h_{i} \in W$, i.e., $\mathcal{I}_{S}=\bigcup_{i=1}^{m} f\left(h_{i}\right)$. Otherwise, the probabilities of the unwitnessed instances in $\mathcal{I}_{S}$ will not be counted when computing the SP of $S$. It then follows that each separable instance of $S$ must be separated by some $h_{i} \in W$. We prove that, in the worst case, we always need $\Omega\left(n N^{d-1}\right)$ hyperplanes to separate all the separable instances of $S$, which implies an $\Omega\left(n N^{d-1}\right)$ lower bound on the runtime of any witness-based SP computing algorithm. We say a hyperplane set $H$ covers a bichromatic dataset $T=T_{R} \cup T_{B}$ iff for any non-trivial separable subset $V \subseteq T$ (i.e., $V$ contains at least one red point and one blue point), there exists $h \in H$ that separates $V$. The following theorem completes the discussion, and is also of independent interest.

- Theorem 5. For a bichromatic dataset $T$, define $\chi(T)$ to be the size of the smallest hyperplane set that covers $T$. Let $\mathcal{T}_{n, N}^{d}$ be the collection of all the bichromatic datasets in $\mathbb{R}^{d}$ containing $n$ red points and $N$ blue points $(n \leq N)$. Define

$$
\Gamma_{d}(n, N)=\sup _{T \in \mathcal{T}_{n, N}^{d}} \chi(T) .
$$

Then for any constant $d$, we have $\Gamma_{d}(n, N)=\Omega\left(n N^{d-1}\right)$. 


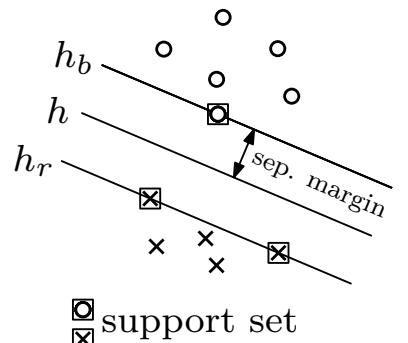

Figure 5 An example in $\mathbb{R}^{2}$.

\section{Expected separation-margin}

In this section, we discuss how to compute the expected separation-margin (ESM) of a stochastic dataset $S=S_{R} \cup S_{B}$. Again, we only describe the details of our algorithm under the unipoint model. The generalization to the multipoint model is straightforward and can be found in the full version [18]. We assume that $S$ has (conventional) general position property.

\subsection{Definitions}

Let $T=T_{R} \cup T_{B}$ be a separable bichromatic dataset and $h$ be a separator. We define the margin of $h$ w.r.t. $T$ as $M_{h}(T)=\min _{a \in T} \operatorname{dist}(a, h)$. The separator which maximizes the margin is called the maximum-margin separator and the corresponding margin is called the separation-margin of $T$, denoted by $\operatorname{Mar}(T)$. If $T$ is not separable or if $T_{R}=\emptyset$ or $T_{B}=\emptyset$, we define its separation-margin to be 0 for convenience. The ESM of a stochastic dataset $S=S_{R} \cup S_{B}$ is the expectation of the separation-margin of the existent points.

Theorem 6. For any separable dataset $T=T_{R} \cup T_{B}$ with $T_{R} \neq \emptyset$ and $T_{B} \neq \emptyset$, the maximum-margin separator of $T$ is unique. Furthermore, for any closest pair $(r, b)$ where $r \in \mathcal{C H}\left(T_{R}\right)$ and $b \in \mathcal{C H}\left(T_{B}\right)$, the maximum-margin separator of $T$ is the bisector of the segment $\overline{r b}$.

Let $h$ be the maximum-margin separator of $T$ and $M=\operatorname{Mar}(T)$ be its separation-margin. Define $C_{R}=\left\{r \in T_{R}: \operatorname{dist}(r, h)=M\right\}$ and $C_{B}=\left\{b \in T_{B}: \operatorname{dist}(b, h)=M\right\}$. We call $C=C_{R} \cup C_{B}$ the support set of $T$ and the points in it the support points. All the support points have the same distance to the maximum-margin separator. Thus, there should exist two parallel hyperplanes $h_{r}$ and $h_{b}$ (both parallel to the maximum-margin separator) where $h_{r}$ goes through all the red support points and $h_{b}$ goes through all the blue ones. We call $h_{r}$ and $h_{b}$ the support planes of $T$. Including the maximum-margin separator $h$, they form a group of three parallel and equidistant hyperplanes $\left(h_{r}, h, h_{b}\right)$. (See Figure 5.) Since the maximum-margin separator is unique, the support set and support planes are also unique. We shall show that the maximum-margin separator can be uniquely determined via the support set.

Theorem 7. Suppose $C$ is the support set of $T$. Then $T$ and $C$ share the same maximummargin separator (also the same separation-margin) and the support set of $C$ is just itself. 


\subsection{Computing the expected separation-margin}

According to Theorem 7, the separation-margin of a separable dataset is equal to that of its support set. Thus, the ESM of $S$ can be computed as

$$
\operatorname{Emar}(S)=\sum_{C \subseteq S}\left(\xi_{S}(C) \cdot \operatorname{Mar}(C)\right)
$$

where $\xi_{S}(C)$ is the probability that the existent points in $S$ are separable with the support set $C$. Since $S$ has the general position property, the size of the support set of the existent points can be at most $2 d$ ( $d$ red points and $d$ blue points at most). It follows that the total number of the possible $C$ to be considered is bounded by $O\left(n^{d} N^{d}\right)$. Indeed, we can further improve this bound.

- Theorem 8. For a given stochastic dataset $S$ with general position property, the total number of the possible support sets is bounded by $O\left(n N^{d}\right)$. As a result, the number of the (distinct) possible separation-margins is also bounded by $O\left(n N^{d}\right)$.

Main proof idea. It is clear that we only need to bound the number of the possible support sets of sizes larger than $d$. We first arbitrarily label all the points in $S$ from 1 to $(n+N)$. For any $C \subseteq S$ with $|C|>d$, we define the representation of $C$ as the $(d+1)$ points in $C$ with the smallest labels (we say those $(d+1)$ points represent $C$ ). Let $a_{1}, a_{2}, \ldots, a_{d+1}$ be a tuple of $(d+1)$ points in $S$ where $a_{1}, \ldots, a_{k}$ are red and $a_{k+1}, \ldots, a_{d+1}$ are blue. If $k=0$ or $k=d+1$, there is no possible support set represented by these $(d+1)$ points because the number of the blue/red points in the support set can at most be $d$. Now consider the case that $1 \leq k \leq d$. It is easy to see that there exist a unique pair of parallel hyperplanes $\left(h_{r}, h_{b}\right)$ such that $h_{r}$ goes through $a_{1}, \ldots, a_{k}$ and $h_{b}$ goes through $a_{k+1}, \ldots, a_{d+1}$, since $S$ is in general position. If $C$ is a possible support set represented by $a_{1}, a_{2}, \ldots, a_{d+1}$, then $h_{r}$ and $h_{b}$ must be the corresponding support planes. That means all the red/blue points in $C$ must lie on $h_{r} / h_{b}$. Note that there are at most $2 d$ points on $h_{r}$ and $h_{b}$, which implies that the number of such $C$ is constant. Consequently, the total number of the possible support sets of $S$ is bounded by $O\left(n N^{d}\right)$. Since the separation-margin is uniquely determined by the support set, the number of the possible separation-margins is also bounded by $O\left(n N^{d}\right)$.

By applying the previous formula for $\operatorname{Emar}(S)$, we can enumerate all the $O\left(n N^{d}\right)$ possible support sets to compute the ESM of $S$. The $O\left(n N^{d}\right)$ possible support sets can be enumerated as follows. For the ones of the sizes less than $(d+1)$, we enumerate them in the obvious way. For the ones of sizes larger than or equal to $(d+1)$, we first enumerate a tuple of $(d+1)$ points (of which at least one is red and one is blue), which would be the representation of the support sets (see the main proof idea of Theorem 8 above). Via these $(d+1)$ points, we can determine two parallel hyperplanes $h_{r}$ and $h_{b}$ where $h_{r}$ goes through the red ones and $h_{b}$ goes through the blue ones. We then find all the points on $h_{r}$ and $h_{b}$, the number of which is at most $2 d$ (including the original $(d+1)$ points). Once we have those points, we are able to enumerate all the possible support sets represented by the original $(d+1)$ points. For each such possible support set $C, \operatorname{Mar}(C)$ can be straightforwardly computed in constant time since $|C| \leq 2 d$. To compute $\xi_{S}(C)$, we observe that $C$ is the support set of the existent points iff

1. all the points in $C_{R}$ (resp., $C_{B}$ ) lie on $h_{r}$ (resp., $h_{b}$ );

2. all the points in $C$ exist;

3. none of the red (resp., blue) points on the same side of $h_{r}$ (resp., $h_{b}$ ) w.r.t. $h$ exists;

4. except the points in $C$, none of the red (resp, blue) points on $h_{r}$ (resp., $h_{b}$ ) exists. 


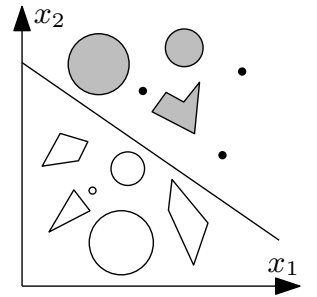

Figure 6 A separability problem for a set of bichromatic general objects in $\mathbb{R}^{2}$.

Among the four conditions, the first one has nothing to do with the existences of the stochastic points. If the enumerated set, $C$, violates this condition, then $\xi_{S}(C)=0$. Otherwise, $\xi_{S}(C)$ is just equal to the product of the existence probabilities of the points in $C$ (the second condition) and the non-existence probabilities of those points which should not exist (the last two conditions). If we use the simplest way, i.e., scanning all the points in $S$, to find the points on $h_{r}$ and $h_{b}$ (for enumerating the possible support sets represented by a set of $(d+1)$ points) as well as to compute each $\xi_{S}(C)$, the total time for computing $\operatorname{Emar}(S)$ is $O\left(n N^{d+1}\right)$. In fact, the runtime can be improved to $O\left(n N^{d}\right)$ by applying tricks similar to the ones used previously for improving the efficiency of our SP computing algorithm. See the full version [18] for details.

\subsection{Hardness of computing expected separation-margin}

We show that the bound achieved in Theorem 8 is tight, which suggests that our algorithm for computing ESM may be difficult to improve further.

- Theorem 9. For any stochastic dataset $S$, define $\kappa(S)$ to be the total number of its (distinct) possible separation-margins. Then for any constant d, there exists some dataset $S$ containing $n$ red points and $N$ blue points $(n \leq N)$ in $\mathbb{R}^{d}$ with $\kappa(S)=\Theta\left(n N^{d}\right)$.

From the above theorem, we can conclude that any algorithm that explicitly considers every possible separation-margin of the stochastic dataset requires at least $\Omega\left(n N^{d}\right)$ time to compute the ESM. This then implies that our algorithm is optimal among this category of algorithms. To do better, the only hope is to avoid considering every possible separationmargin explicitly. However, this is fairly difficult (though may not be impossible) because of the lack of an explicit relationship among distinct separation-margins.

\section{Extension to general geometric objects}

In the previous sections, we considered the separability related problems for stochastic points only. In fact, the two problems can be naturally generalized to the case of general stochastic geometric objects (see Figure 6). In this paper, the general objects to be considered include polytopes with constant number of vertices, and/or $d$-dim closed balls with various radii. We show that, with some effort, our methods can be extended to solve the generalized versions of the SP and ESM problems. The stochastic model used is similar to the unipoint model: each object has a fixed location with an associated existence probability. For convenience, we still use $S=S_{R} \cup S_{B}$ to denote the given stochastic dataset, in which each element is either a polytope or a ball. 


\subsection{Reducing polytopes to points}

To deal with polytopes is easy, because of the fact that the entire polytope is on one side of a (hyperplane) separator iff all its vertices are. Thus, we can simply replace each polytope with its vertices and associate with each vertex an existence probability equal to that of the polytope. In this way, the polytopes in $S$ can be reduced to points. One thing should be noted is that, once we reduce the polytopes to points, the existences of the vertices of each polytope are dependent upon each other. However, this issue can be easily handled without any increase in time complexity, because each polytope only has $O(1)$ vertices.

\subsection{Handling balls}

Once we are able to use the vertices to replace the polytopes, it suffices to consider the separability problems for datasets containing only stochastic balls (points can be regarded as 0-radius balls). Before we discuss how to handle balls, we need a definition of general position for a ball-dataset. We say a set of balls in $\mathbb{R}^{d}$ is in general position (or has the general position property) if

1. the centers of the balls are in general position;

2. no $(d+1)$ balls have a common tangent hyperplane.

Furthermore, we say a ball-dataset has strong general position property (SGPP) if it satisfies the two conditions above and all of the 0-radius balls in it have SGPP (as defined in Section 2) when regarded as points. In Section 4.2.1, the given ball-dataset $S$ is required to have SGPP. In Section 4.2.2, we only need the assumption that $S$ has the (usual) general position property.

\subsubsection{Separable-probability (ball-version)}

Let $T=T_{R} \cup T_{B}$ be a set of bichromatic balls with SGPP and set $J=\{3,4, \ldots, d\}$. Theorem 1 and 2 can be easily generalized to ball-datasets (the meaning of $\mathcal{C H}\left(T_{R}\right) / \mathcal{C H}\left(T_{B}\right)$ should be modified as the convex hull of all the balls in $T_{R} / T_{B}$ ). The ball-version of Theorem 3 (and also its proof) is slightly different, which we present as follows (the proof can be found in the full version [18]).

- Theorem 10. $T^{U^{*}}$ is weakly separable and there exists only one weak separator. Furthermore, the unique weak separator of $T^{U^{*}}$ either goes through exactly d 0-radius balls (of which at least one is in $T_{R}^{U^{*}}$ and one is $T_{B}^{U^{*}}$ ) or is tangent to at least one ball with radius larger than 0 .

Once we generalize these results, we are immediately able to generalize the concept of extreme separator to ball-datasets. As we do in Section 2.1, if $P_{0} \neq \emptyset$, we define the extreme separator of $T$ as the derived separator of the unique weak separator of $T^{U^{*}}$. If $P_{0}=\emptyset$, we recursively define the extreme separator of $T$ as the derived separator of the extreme separator of $\Phi_{J}(T)$. If the extreme separator is directly defined (i.e., the case of $P_{0} \neq \emptyset$ ), we call the subset consisting of all the balls tangent to extreme separator the critical set. We shall use the following theorem later for solving the ball version of the SP problem.

- Theorem 11. Let $T=T_{R} \cup T_{B}$ be a separable ball-dataset whose extreme separator is directly defined and let $C$ be its critical set. Then the extreme separator of $C$ is also directly defined. Furthermore, $T$ and $C$ share the same extreme separator and auxiliary subspace.

Theorem 11 implies that the extreme separator is uniquely determined by the critical set. This then gives us the basic idea to solve the problem, enumerating all the possibilities for 
the critical set. As in Section 2.2, we can compute the SP of $S$ as

$$
\operatorname{Sep}(S)=\operatorname{Sep}\left(\Phi_{J}(S)\right)+\sum_{C \subseteq S} \lambda_{S}(C)
$$

where $\lambda_{S}(C)$ is the probability that the critical set of the existent balls is $C$. Since the balls in $S$ have SGPP, the size of the critical set of the existent balls can be at most $d$. Furthermore, the critical set should contain at least one red ball and one blue ball. Thus, it suffices to compute $\lambda_{S}(C)$ for all the subsets $C \subseteq S$ with $|C| \leq d$ which contain balls of both colors. We consider two cases separately. First, all the balls in $C$ have radius 0 . Second, there is at least one ball in $C$ with radius larger than 0 .

In the first case, according to Theorem $10, \lambda_{S}(C)>0$ only if $|C|=d$. Since the balls in $C$ are actually points, the situation here is almost the same as what we confronted in the point-version of the problem. We can uniquely determine a hyperplane $h$ which goes through the $d$ points in $C$, and a subspace $U^{*} \in \mathcal{V}$ perpendicular to $h$. Then $\lambda_{S}(C)$ is just equal to the probability that $h$ is the extreme separator of the existent balls. Also, the conditions for $h$ to be the extreme separator are very similar to those in Section 2.2, which are

1. all the balls in $C$ exist;

2. there exist $r \in \mathcal{C H}\left(C_{R}\right)$ and $b \in \mathcal{C H}\left(C_{B}\right)$ such that their projection images on $U^{*}$ coincide;

3. no red (resp. blue) ball on the opposite (resp. same) side of $h$ w.r.t. the point $o$ exists, where the definition of $o$ is similar to that in Section 2.2;

4. no ball intersecting with $h$ exists, except the ones in $C$.

If $C$ violates the second condition, then $\lambda_{S}(C)=0$. Otherwise, $\lambda_{S}(C)$ is just equal to the product of the existence probabilities of the balls in $C$ and the non-existence probabilities of the balls that should not exist.

In the second case, however, the size of $C$ may be less than $d$. According to Theorem 11, if $C$ is the critical set of the existent points, then the extreme separator and auxiliary subspace of the existent points are the same as those of $C$. This implies that $\lambda_{S}(C)=0$ if $C$ is not separable or the extreme separator of $C$ is defined recursively. So we only need to consider the situation that the extreme separator of $C$ is directly defined. Assume that $C$ has the extreme separator $h$ (directly defined) with the auxiliary subspace $U^{*} \in \mathcal{V}$. Let $c$ be any ball in $C$ with radius larger than 0 . Then it is easy to see that $C$ is the critical set of the existent balls iff

1. all the balls in $C$ exist;

2. all the balls in $C$ are tangent to $h$;

3. no ball with the same color as (resp. different color than) $c$ but on the opposite (resp. same) side of $h^{*}$ w.r.t. $c$ exists;

4. no ball intersecting with $h$ exists, except the ones in $C$.

Because $|C|=O(1), h$ and $U^{*}$ can be computed in constant time using brute-force. Similarly, if $C$ satisfies the second condition, $\lambda_{S}(C)$ is equal to the product of the existence probabilities of the balls in $C$ and the non-existence probabilities of the balls that should not exist.

In both the cases, $\lambda_{S}(C)$ can be computed in linear time by simply scanning all the balls in $S$. Thus, the SP can be finally computed in $O\left(n N^{d}\right)$ time, as the number of the subsets $C$ considered is bounded by $O\left(n N^{d-1}\right)$. Unfortunately, the improvement techniques used in the point-version of the problem cannot be generalized to ball-datasets so that our eventual time bound for computing the SP of general geometric objects remains $O\left(n N^{d}\right)$. 


\subsubsection{Expected separation-margin (ball-version)}

Let $T=T_{R} \cup T_{B}$ be a set of bichromatic balls in general position. Clearly, the definitions given in Section 3.1 (maximum-margin separator, separation-margin, support set/points/planes, etc.) can be directly generalized to ball-datasets. Also, with these definitions, the ball-versions of Theorem 6 and 7 can be easily verified (by using the same proofs).

To extend the previous algorithm, we need to prove the ball version of Theorem 8 . The first step is the same as that in the original proof of Theorem 8: we arbitrarily label the balls in $S$ and define the representation of $C$ as the $(d+1)$ balls in $C$ with the smallest labels, for any $C \subseteq S$ with $|C|>d$. We show that the number of possible support sets represented by any group of $(d+1)$ balls is $O(1)$. Let $a_{1}, a_{2}, \ldots, a_{d+1}$ be any $(d+1)$ balls in $S$ where $a_{1}, \ldots, a_{k}$ are red and $a_{k+1}, \ldots, a_{d+1}$ are blue, where $1 \leq k \leq d$ as before. Let each ball $a_{i}$ have center $c_{i}$ and radius $\delta_{i}$. If some possible support set $C$ is represented by these $(d+1)$ balls, then the support plane $h_{r}$ (resp. $\left.h_{b}\right)$ must be tangent to $a_{1}, \ldots, a_{k}$ (resp. $\left.a_{k+1}, \ldots, a_{d+1}\right)$. Furthermore, the balls $a_{1}, \ldots, a_{k}$ (resp. $\left.a_{k+1}, \ldots, a_{d+1}\right)$ must be on the open side of $h_{r}$ (resp. $h_{b}$ ), i.e., the side different from the one containing the area between $h_{r}$ and $h_{b}$. It can be shown that there are at most two possibilities for the support planes $\left(h_{r}, h_{b}\right)$ (see the full version [18]). Then by following the logic in the proof of Theorem 8, we know the number of the possible support sets represented by these $(d+1)$ balls is constant, which immediately implies that the total number of all possible support sets is $O\left(n N^{d}\right)$.

To enumerate these possible support sets, we can directly use the same method as in Section 3.2, i.e., first enumerate $(d+1)$ balls and then enumerate the possible support sets represented by them. Again, because the improvement techniques used in the point-version of the problem do not work for ball-datasets, we have to scan all the balls once for computing the corresponding probability of each possible support set, which makes the overall time $O\left(n N^{d+1}\right)$ for computing the ESM of general geometric objects.

\section{Applications}

In this section, we present some applications of our algorithms to stochastic convex-hull (SCH) related problems. Given a stochastic (point) dataset $A$, the $\mathrm{SCH}$ of $A$ refers to the convex-hull of the existent points in $A$, which is an uncertain convex shape.

\subsection{SCH membership probability problem}

The SCH membership probability problem was introduced for the first time in [4]. The problem can be described as follows: given a stochastic dataset $A=\left\{a_{1}, \ldots, a_{m}\right\} \subset \mathbb{R}^{d}$ and a query point $q \in \mathbb{R}^{d}$, compute the probability that $q$ is inside the SCH of $A$, which we call the $S C H$ membership probability (SCHMP) of $q$ w.r.t. $A$.

It is shown in [11] that the SCHMP problem in $\mathbb{R}^{d}$ can be reduced to the SP problem in $\mathbb{R}^{d-1}$. Due to this, by plugging in our SP computing algorithm presented in Section 2 , we immediately obtain an $O\left(m^{d-1}\right)$-time algorithm to compute SCHMP for $d \geq 3$, which matches the best known bound in [11]. Indeed, this bound can be achieved by applying any SP computing algorithm with runtime bounded by $O\left(N^{d}\right)$.

More interestingly, we show that our SP computing algorithm yields a more direct and natural method to solve the SCHMP problem in $O\left(m^{d-1}\right)$ time for $d \geq 3$ and $O(m \log m)$ time for $d=2$, which does not involve any non-trivial reduction between the two problems. Given a SCHMP problem instance $(A, q)$, clearly, the query point $q$ is outside the SCH of $A$ iff it can be separated from the existent points in $A$ by a hyperplane. Thus, we construct 
a stochastic bichromatic dataset $S=S_{R} \cup S_{B}$, where $S_{R}$ contains only one point, $q$, with existence probability 1 and $S_{B}=A$. Then the SCHMP of $q$ w.r.t. A is just equal to $1-\operatorname{Sep}(S)$. This can be computed in $O\left(m^{d-1}\right)$ time for $d \geq 3$ and $O(m \log m)$ time for $d=2$ by applying our SP computing algorithm, since $\left|S_{R}\right|=1$ and $\left|S_{B}\right|=m$. Note that the $O\left(m^{d-1}\right)$ runtime of this simple method relies on the $O\left(n N^{d-1}\right)$ time bound of our SP computing algorithm for $d \geq 3$. If we plug in an $O\left(N^{d}\right)$-time SP computing algorithm, the time cost will become $O\left(m^{d}\right)$. Interestingly enough, this method for computing SCHMP is a generalization of the witness-edge method in [4] to the case $d>2$, where the latter was the first known approach that solves this problem in $\mathbb{R}^{2}$ and was thought to be difficult to be generalized to higher dimensions [4]. This can be seen as follows. When plugging in our SP computing algorithm, we enumerate all the possible extreme separators of $\{q\} \cup \Gamma$, where $\Gamma$ denotes the set of the existent points in $A$. If the extreme separator is finally defined in $\mathbb{R}^{d-2 k}$, it goes through $(d-2 k)$ points, of which one is $q$. These $(d-2 k)$ points form a $(d-2 k-1)$-dim face of $\mathcal{C H}(\{q\} \cup \Gamma)$ about the vertex $q$. It is evident that this face is uniquely determined by the convex polytope $\mathcal{C H}(\{q\} \cup \Gamma)$. We call it the witness-face of $q$ in $\mathcal{C H}(\{q\} \cup \Gamma)$. Then enumerating the possible extreme separators is equivalent to enumerating the possible witness-faces of $q$ in $\mathcal{C H}(\{q\} \cup \Gamma)$. When $d=2$, the concept of witness-face coincides with that of witness-edge defined in [4]. Thus, in this case, our method is identical to the witness-edge method.

\subsection{Other SCH-related problems}

Our algorithms presented in the previous sections can also be applied to solve some new problems related to $\mathrm{SCH}$. Here we propose three such problems and show how to solve them.

- SCH intersection probability problem. This problem is a natural generalization of the SCHMP problem. Given a stochastic dataset $A=\left\{a_{1}, \ldots, a_{m}\right\} \subset \mathbb{R}^{d}$ and a query object $Q$ which is a convex polytope with constant complexity (e.g., segment, simplex, etc.) in $\mathbb{R}^{d}$, the goal is to compute the probability that $Q$ has non-empty intersection with the $\mathrm{SCH}$ of $A$. When $Q$ is a single point, this is just the SCHMP problem. To solve this problem, we extend the method described in the preceding subsection. According to Theorem 1, $Q$ has no intersection with the $\mathrm{SCH}$ iff its vertices can be separated from the existent points in $A$ by a hyperplane. Based on this, by constructing a stochastic bichromatic dataset $S=S_{R} \cup S_{B}$, where $S_{R}$ contains the vertices of $Q$ with existence probability 1 and $S_{B}=A$, we can apply our SP computing algorithm to compute the desired probability in $O\left(m^{d-1}\right)$ time (note that $\left|S_{R}\right|$ is $O(1)$ for $Q$ has constant complexity).

- SCH $\varepsilon$-distant probability problem. This problem is another natural generalization of the SCHMP problem. Given a stochastic dataset $A=\left\{a_{1}, \ldots, a_{m}\right\} \subset \mathbb{R}^{d}$, a query point $q \in \mathbb{R}^{d}$, and a parameter $\varepsilon \geq 0$, the goal is to compute the probability that the distance from $q$ to the $\mathrm{SCH}$ of $A$ is greater than $\varepsilon$. When $\varepsilon=0$, this is equivalent to the SCHMP problem. To solve this problem, we need to apply our generalized SP computing algorithm presented in Section 4. Clearly, $q$ has a distance greater than $\varepsilon$ to the SCH of $A$ iff the $\varepsilon$-ball centered at $q$ can be separated from the existent points in $A$ by a hyperplane. Thus, by constructing a generalized stochastic bichromatic dataset $S=S_{R} \cup S_{B}$, where $S_{R}$ contains the $\varepsilon$-ball centered at $q$ with existence probability 1 and $S_{B}=A$, we can apply our generalized SP computing algorithm to compute the desired probability in $O\left(m^{d}\right)$ time.

- Expected distance to a SCH. Given a stochastic dataset $A=\left\{a_{1}, \ldots, a_{m}\right\} \subset \mathbb{R}^{d}$ and a query point $q \in \mathbb{R}^{d}$, the goal of this problem is to compute the expected distance from $q$ to the $\mathrm{SCH}$ of $A$. To achieve this, we notice that the distance from $q$ to the $\mathrm{SCH}$ of $A$ is 
just equal to the separation-margin of $\{q\} \cup \Gamma$, where $\Gamma$ denotes the set of the existent points in $A$. Thus, we construct a stochastic bichromatic dataset $S=S_{R} \cup S_{B}$, where $S_{R}$ contains only one point $q$ with existence probability 1 and $S_{B}=A$. Then the problem can be solved in $O\left(\mathrm{~m}^{d}\right)$ time by plugging in our ESM computing algorithm presented in Section 3 .

\section{References}

1 P.K. Agarwal, B. Aronov, S. Har-Peled, J.M. Phillips, K. Yi, and W. Zhang. Nearest neighbor searching under uncertainty II. In Proceedings of the 32nd Symposium on Principles of Database Systems, pages 115-126. ACM, 2013.

2 P.K. Agarwal, S-W. Cheng, Y. Tao, and K. Yi. Indexing uncertain data. In Proceedings of the Twenty-eighth ACM SIGMOD-SIGACT-SIGART Symposium on Principles of Database Systems, pages 137-146. ACM, 2009.

3 P.K. Agarwal, S-W. Cheng, and K. Yi. Range searching on uncertain data. ACM Transactions on Algorithms (TALG), 8(4):43, 2012.

4 P.K. Agarwal, S. Har-Peled, S. Suri, H. Ylldı, and W. Zhang. Convex hulls under uncertainty. In Algorithms-ESA 2014, pages 37-48. Springer, 2014.

5 C.C. Aggarwal and P.S. Yu. A survey of uncertain data algorithms and applications. IEEE Transactions on Knowledge and Data Engineering, 21(5):609-623, 2009.

6 A. Agrawal, Y. Li, J. Xue, and R. Janardan. The most-likely skyline problem for stochastic points. Submitted to a journal, 2015.

7 N. Dalvi, C. Ré, and D. Suciu. Probabilistic databases: diamonds in the dirt. Communications of the ACM, 52(7):86-94, 2009.

8 M. de Berg, A.D. Mehrabi, and F. Sheikhi. Separability of imprecise points. In Algorithm theory - SWAT 2014, volume 8503 of LNCS, pages 146-157. Springer, 2014.

9 M. de Berg, M. van Kreveld, M. Overmars, and O.C. Schwarzkopf. Computational Geometry. Springer, 2000.

10 H. Edelsbrunner and L.J. Guibas. Topologically sweeping an arrangement. In Proceedings of the Eighteenth Annual ACM Symposium on Theory of Computing, pages 389-403, 1986.

11 M. Fink, J. Hershberger, N. Kumar, and Suri S. Hyperplane separability and convexity of probabilistic points. 32nd International Symposium on Computational Geometry, 2016.

12 P. Kamousi, T.M. Chan, and S. Suri. Stochastic minimum spanning trees in euclidean spaces. In Proceedings of the Twenty-seventh Annual Symposium on Computational geometry, pages 65-74. ACM, 2011.

13 Y. Li, J. Xue, A. Agrawal, and R. Janardan. On the arrangement of stochastic lines in $\mathbb{R}^{2}$. Submitted to a journal, 2015.

14 M. Löffler. Data imprecision in computational geometry. PhD thesis, Utrecht Univ., 2009.

15 M. Löffler and M. van Kreveld. Largest and smallest convex hulls for imprecise points. Algorithmica, 56(2):235-269, 2010.

16 S. Suri and K. Verbeek. On the most likely Voronoi Diagram and nearest neighbor searching. In Algorithms and Computation, pages 338-350. Springer, 2014.

17 S. Suri, K. Verbeek, and H. Yıldız. On the most likely convex hull of uncertain points. In Algorithms-ESA 2013, pages 791-802. Springer, 2013.

18 J. Xue, Y. Li, and R. Janardan. On the separability of stochastic geometric objects, with applications. (Full version). ArXiv:1603.07021 [cs.CG], 2016.

19 W. Zhang. Nearest-neighbor searching under uncertainty. PhD thesis, Duke Univ., 2012. 